\title{
The Relationship Between Recurrent Abdominal Pain and Helicobacter Pylori Infection in Children Miky Akbar ${ }^{\mathrm{a}}$, Supriatmo ${ }^{\mathrm{b}}$, Winra Pratita ${ }^{\mathrm{a}, \mathrm{b}}$
}

mikyakbar@gmail.com

Resident of Department of Child Health, Medical School, Universitas Sumatera Utara/Adam Malik Hospital, Medan bGastroenterology Consultant of Department of Child Health, Medical School, Universitas Sumatera Utara/Adam Malik Hospital, Medan

\begin{abstract}
This study aimed to determine the relationship between recurrent abdominal pain and Helicobacter pylori infection in children. The cross sectional study was conducted 56 children. Subjects were children between aged 2-18 years with complaints of abdominal pain based on the Rome IV criteria according to the inclusion and exclusion criteria. Interview was carried out to establish the diagnosis of recurrent abdominal pain. Helicobacter pylori infection was determined based on Helicobacter Pylori Stool Antigen (HPSA) test measurements.From 56 samples, there were 36 children with positive HPSA and 20 children with negative HPSA. Symptoms of abdominal pain occurred in 16 children (44.4\%) in the HPSA positive group, while in the HPSA negative group all had abdominal pain. There was a significant association between abdominal pain symptoms and $\mathrm{H}$. pylori infection $(\mathrm{p}<0.001)$.
\end{abstract}

Published by IJRP.ORG. Selection and/or peer-review under responsibility of International Journal of Research Publications (IJRP.ORG)

Keyword : Recurrent abdominal pain, Helicobacter pylori, child.

\section{Introduction}

H.pylori bacterial infection is one of the most common infections in the world and is associated with the risk of various stomach disorders. This bacterium was discovered in the stomach by Warren and Marshall in 1982 and has began new insights into the causes of upper gastrointestinal disorders (Hegar, B., 2000). The prevalence of $\mathrm{H}$. pylori in developing countries is reported to be higher than in developed countries. The prevalence of H.pylori in children in developed countries is estimated at $10 \%$, while in developing countries it is estimated to be between $30-80 \%$. The prevalence in Asia was reported from $8.2 \%$ to $11 \%$, whereas in Jakarta, the prevalence of H.pylori infection based on serological examinations in 150 elementary school students was $27 \%$ and $90 \%$ of those who had seropositive found H.pylori in their stomach (Uwan et al., 2016). Abdominal pain in children is a very common symptom of most school-aged children. Most children with complaints of abdominal pain are considered normal by their parents and are not taken to a doctor's consultation, this can cause recurrent abdominal pain. Recurrent abdominal pain (RAP) in children has been considered by some investigators to be a clinical symptom associated with H.pylori infection. Data from several studies show that $22-37 \%$ of children with recurrent abdominal pain are shown to have H.pylori infection by serological examination. Other complaints often mentioned are pain in the epigastric area, waking up at night, and vomiting (Plunkett., 2005). Complaints of recurrent abdominal pain in children can be assessed based on Roman criteria. The Rome criteria were first issued in 1989, and the latest in 2016 is the Rome IV. The criteria to diagnose H. pylori infection in children can be done by invasive or non-invasive methods . Examination Helicobacter Pylori Stool Antigen (HPSA) is one of the non-invasive methods that is 
easy to do and cost-effective (Giuseppina., 1998). Research by Ali mohammadi et al in 2016 showed as many as 145 children with recurrent abdominal pain complaints, 85 of whom showed positive HPSA examination results (58.6\%) (Devanaraya et al., 2017). The increasing number of children experiencing complaints of atypical abdominal pain and the lack of research on H.pylori conducted in Medan, is the basis of this research.

\section{Methods}

\subsection{Subjects and Methods}

This was a cross sectional study to examine the relationship between abdominal pain and H.pylori infection in children. This study conducted among 56 children at RSUP. H. Adam Malik Medan and University Sumatera Utara Hospital between September 2019 until June 2020. The target population in this study was children between two until eighteen years old. The sample in this study is the population who met inclusion and exclusion criterias. Inclusion criterias were children between two until eighteen years old, experiencing recurrent abdominal pain based on Rome IV criteria, showing the results of the Helicobacter Pylori Stool Antigen (HPSA) examination, agreeing and signing an informed consent form. The exclusion criteria were history of drug use such as proton pump inhibitors (PPIs), H2 blocker antagonists, antibiotics and non-steroidal anti-inflammatory drugs (NSAIDs) in the past 14 days, as well as patients who had a history of gastric surgery, gastric bleeding, cirrhosis of the liver, kidney failure requiring dialysis, heart failure, gastric cancer. After the interview, subjects were divided into two groups, recurrent abdominal pain and did not experience recurrent abdominal pain, then conducted HPSA test so that it can be seen the relationship of recurrent abdominal pain with Helicobacter Pylori infection in children. Statistical assessment was performed using the Chi square test.

\subsection{Research Ethics}

This study was approved by the Ethics Committee of the Faculty of Medicine, Universitas Sumatera Utara / Haji Adam Malik General Hospital in Medan No: 957/TGL/KEPK FK USU-RSUP HAM/2019.

\subsection{Statistical Analysis}

Data was statistically analyzed using SPSS software version 23.0 (Statistical Package for Social Sciences) for windows. Descriptive statistics were expressed in the form of mean \pm standard deviation (SD) for normally distributed data and median/range test for the not normally distributed data; while for categorical data they were presented in the form of frequency. Association between variables were performed by chi square test with $\mathrm{p}$ value of $(<0.05)$ was considered as a significant difference.

\section{Results}

56 subjects fulfill the inclusion and exclusion criterias. From all the subjects, there were 22 male subjects $(39.3 \%), 34$ female subjects $(60.7 \%)$. The age of the subjects has a median at 12,7 years. Through anthropometric measurements, the body weight has a median at 35,5 (SD 12,95) $\mathrm{kg}$. The mean height of subjects was 135,98 (SD 23,67) cm. Assessment of body weight according to height found 13 subjects $(23,2 \%)$ were overweight, 34 subjects $(60.7 \%)$ were normal and 9 subjects $(16 \%)$ were malnutrition. Thirty six subjects $(64.3 \%)$ suffered recurrent abdominal pain based on the criteria of Rome IV and 20 subjects $(35.7 \%)$ did not suffered recurrent abdominal pain (Table 1). 
Table 1 Characteristics of Research Subjects $(n=56)$

\begin{tabular}{|c|c|}
\hline Subject Characteristics & $\mathrm{n}=56$ \\
\hline \multicolumn{2}{|l|}{ Gender, n (\%) } \\
\hline Male & $22(39.3)$ \\
\hline Women & $34(60.7)$ \\
\hline \multicolumn{2}{|l|}{ Age, years } \\
\hline Mean & 12.07 \\
\hline Median & 12.9 \\
\hline $\mathrm{SD}$ & 3.43 \\
\hline Min - mom & $3,2-17,80$ \\
\hline \multicolumn{2}{|l|}{ Weight, kg } \\
\hline Mean & 37.72 \\
\hline Median & 35.5 \\
\hline SD & 12.95 \\
\hline Min - mom & $9,10-85$ \\
\hline \multicolumn{2}{|l|}{ Height, cm } \\
\hline Mean & 135.98 \\
\hline Median & 143 \\
\hline $\mathrm{SD}$ & 23.67 \\
\hline Min - mom & $13.8-168$ \\
\hline $\begin{array}{l}\text { Nutritional status, n (\%) } \\
\text { Overweight } \\
\text { Good nutrition } \\
\text { Malnutrition }\end{array}$ & $\begin{array}{l}13(23.2) \\
34(60.7) \\
9(16)\end{array}$ \\
\hline \multicolumn{2}{|c|}{ Recurrent Abdominal pain, $\mathrm{n}(\%$} \\
\hline Yes & $36(64.3)$ \\
\hline No & $20(35.7)$ \\
\hline
\end{tabular}

The results of the HPSA examination showed that from 56 subjects of the study, there were 36 children with $\mathrm{H}$. Pylori infection (HPSA positive) and 20 children without H. pylori infection (HPSA negative). Symptoms of abdominal pain occurred in 16 child subjects $(44.4 \%)$ of the group with H. pylori infection while in the group without H. pylori infection, all of them had abdominal pain $(\mathrm{p}<0.001)$ (Table 2). The proportion of the children with and without H. Pylori infection is further presented in Figure 1.

Table 2 Relationship between recurrent abdominal pain and $\mathrm{H}$. Pylori incidence

\begin{tabular}{llll}
\hline Recurrent abdominal Pain & \multicolumn{2}{c}{ H. pylori } & $\mathrm{p}$ \\
\hline Yes & Positive $(\mathrm{n}=32)$ & Negative $(\mathrm{n}=20)$ & $<0.001^{\mathrm{a}}$ \\
\hline No & $16(44.4)$ & $20(100)$ & \\
\hline
\end{tabular}

${ }^{a}$ Fischer's Exact, ${ }^{b}$ Chi Square 


\section{Discussion}

The risk of $\mathrm{H}$. pylori infection is associated with many factors related the host-agent-environment. H. pylori colonization begins early in life. During the time of neonatal source of infection will be finite from caregivers, family members, or babysitter. The growth will increase the exposure to multiple sources of infection, which may evaluate the higher infection rates in children starting school (Švagelj B et al., 2017). Study of Aitila et al, (2019) reported that the prevalence of H.pylori infection was mostly found in children aged 11-15 years and the prevalence of $\mathrm{H}$. pylori infection increases with increasing age from $16.2 \%$ for children aged $1-5$ years, $27.2 \%$ for ages $6-10$ years, and $36.71 \%$ for ages $11-15$ year. In this study, the mean age of children who experienced abdominal pain with H.pylori infection was 12.13 years, this shows that in this study there was an increase in the prevalence of H.pylori infection by $36.71 \%$.

In this study, the prevalence of $\mathrm{H}$. pylori infection was more prevalent in female children as many as 24 children $(66.7 \%)$ compared to male children as many as 12 children $(33.3 \%)$. These results support previous studies in Pontianak which showed that the prevalence of $\mathrm{H}$. pylori infection was higher on women (Uwan et al., 2016). The Roma IV criterion is a standardized scoring system to aid in the diagnosis and management of functional digestive disorders in children. Abdominal pain is the main complaint of gastrointestinal system disorders in preschool and school children with a prevalence of around $10 \%$ (Schmulson et al., 2017; Baalemanet al., 2020) In this study, the prevalence of abdominal pain in children was 28.5\%. Abdominal pain experienced by almost all subjects occurred at least twice in a three month period, accompanied by cold sweats during abdominal pain (Devanaraya et al., 2017). The location of the occurrence of abdominal pain in the subjects in this study was mostly in the periumbilical area and interfered with activities in children. Based on ROME IV criteria, abdominal pain in the subjects corresponded to abdominal migraine (H2c classification).

This study found a significant association between recurrent abdominal pain symptoms and $\mathrm{H}$. pylori infection. A total of 36 children experienced H. Pylori infection through positive HPSA examination, 16 of them (44.4\%) were found with symptoms of recurrent abdominal pain and 20 of them did not. A total of 20 children were found to have a negative HPSA examination, which showed no H.pylori infection but all had symptoms of recurrent abdominal pain. This suggests that abdominal pain in children is not always caused by H.pylori infection. Abdominal pain can occur as a clinical manifestation due to inflammatory reactions mediated by various inflammatory mediators that disrupt the integrity of the stomach cell walls.

A 2013 study in Iran on 103 children who experienced recurrent abdominal pain symptoms, as many as 39 children (37. 8\%) of whom found positive HPSA examination results. This indicates that $37.8 \%$ of children with recurrent abdominal pain were due to H. Pylori infection as an organic cause. A 2013 Nepal study showed that among 47 children with symptoms of abdominal pain, 41 children (87\%) had organic causes and $6(13 \%)$ non-organic causes. Giardiasis was found to be the most common organic cause (46\%) for RAP, followed by chronic constipation (34\%) and urine culture-proven urinary tract infection (7.3\%). Upper gastrointestinal endoscopy performed in six patients showed antral gastritis with esophagitis and with $\mathrm{H}$. pylori infection (Joshi., 2013; Hegar., 2000).

Tests to detect H. Pylori infection are broadly divided into invasive and non-invasive. The gold standard in diagnosing H. pylori infection is endoscopic biopsy of stomach tissue with rapid urease / CLO test, histology, and culture, but it is an invasive procedure that has obstacles as anesthesia, discomfort, and ethical issues. HPSA examination is a non-invasive test to diagnose $\mathrm{H}$. Pylori infection which has a sensitivity and specificity value of $85 \%$ and $93 \%$, respectively. Several previous studies have compared the sensitivity and specificity of HPSA testing with other invasive tests in diagnosing H. pylori infection (Watanabe et al., 2013).

The Iranian study also reported a sensitivity and specificity of HPSA of more than $90 \%$ in children with gastrointestinal symptoms. The use of HPSA in children with abdominal pain in developing countries is still under debate, but the HPSA examination is the optimal examination as a screening and evaluation of success in eradicating H. Pylori infection therapy (Alimohammadi et al., 2016; Iranikhah et al., 2013). In this study, all subjects were examined for HPSA and no endoscopic examination was performed, so that gastritis 
was not included as a cause of abdominal pain in all subjects in this study. The limitation of this study does not take into account on characteristics of abdominal pain and environmental factors related to abdominal pain experienced by subjects. Further research needs to be assessed to associate abdominal pain with Helicobacter Pylori infection in children, especially with the endoscopic examination / CLO test.

\section{Conclusion}

There is a relationship between recurrent abdominal pain and H.pylori infection in children.

\section{Acknowledgements}

The author thanks to all clinical staff of Pediatric Gastroenterology, Universitas Sumatera Utara, who were involved and contributed for the completion of this study.

\section{References}

Aitila P. Mutyaba M. Okeny S. Kasule MN. Kasule R. Ssedyabane F.Prevalence and Risk Factors of Helicobacter pylori Infection among Children Aged 1 to 15 Years at Holy Innocents Children's Hospital, Mbarara, South Western Uganda. Journal of Tropical Medicine. 2019; 20: 1-5.

Alimohammadi H. Fouladi N. Salehzadeh F. Alipour SA. Javadi MS. Childhood recurrent abdominal pain and Helicobacter pylori infection, Islamic Republic of Iran. EMHJ. 2016; 22:12.

Baaleman DF. Lorenzo CD. Marc A. Saps M. The Effects of the Rome IV Criteria on Pediatric Gastrointestinal Practice. Curr Gastroenterol Rep. 2020; 22: 21

Boediarto A, Abdominal pain in children. In: Juffrie M, Oswari H, Soenarto S, editors. Textbook of gastroentero-hepatology. Jakarta: UKK-gastroentero-hepatology IDAI: 2010.149-65.

Briand H, Yin C, Hong W, Rivoiron S. Rapid detection of H. pylori Ag in human stool with the new bioNexia H. pylori Ag test. Europian Helicobacter Microbiota Study Group. 2015

Carin E, Williams A. Recurrent Abdominal Pain in Children. American Family Physician. 2018: 97; 12

Chi H. Bair MJ. Wu MS. Chiu NC. Hsiao YC. Chang KY.Prevalence of Helicobacter pylori Infection in High-school Students on Lanyu Island, Taiwan: Risk Factor Analysis and Effect on Growth. J Formos Med Assoc. 2009; 108: 12.

Devanaraya NM, Rajindrajith S, et al. Recurrent Abdominal Pain in Children. Indian Ped J. 2009;4.

Giuseppina OD, Palli, Saieva C, Chiorboli E, Bona. Short stature and Helicobacter pylori infection in Italian children: prospective multicentre hospital based case control study. BMJ. 1998; 317.

Hegar B, Helicobacter pylori infection in children. Sari Pediatrics: 2000:2:2.

Huang RC, Plamer LJ, Forbes DA. Prevalence and pattern of childhood abdominal pain in an Australian general practice. J Pediatr Child Health. 2000; 36: 349-53

Iranikhah A Ghadir MR. Sarkeshikian S. Saneian H. Heiari A. Mahvari M. Stool Antigen Tests for the Detection of Helicobacter Pylori in Children. Iran J Pediatr. 2013; 23: 2: 138-142.

Joshi BG. Etiology of Recurrent Abdominal Pain in Children. J Nepal Paediatr Soc. 2013; 33 (1): 31-33.

Plunkett A, Beattie RM. Recurrent abdominal pain in childhood. JR Soc Med. 2005; 98: 101-106.

Schmulson MJ dan Drossman DA. What Is New in Rome IV. J Neurogastroenterol Motil. 2017;23(2), p:15163.

Švagelj B, Brkic H, Cvrkovic M, Svagel M, Terzice V, Brkic N. The prevalence and characteristics of Helicobacter pylori-associated gastritis in dyspeptic patients in Eastern Croatia, determined by immunohistochemistry. Period of the viol. 2017; 119: 1.

Uwan WB, Syam AF, Lesmana CRA, Rumende CM. Differences in Prevalence of Helicobacter pylori Infection between Ethnic Chinese and Dayaks with Dyspepsia Syndrome. J Indonesian Internal Medicine. 2016; 3: 1 . 
Miky Akbar / International Journal of Research Publications (IJRP.ORG)

Watanabe K, et al. Accuracy of Endoscopic Diagnosis of Helicobacter Pylori Infection According to Level of

Endoscopic Experience and The Effect of Training. BMC Gastroenterology. 2013; 13: 128. 